\title{
Preparation and Characterization of Poly- $N$-isopropylacrylamide Cryogels containing Liposomes and Their Adsorption Properties of Tryptophan
}

\author{
Yui HIRAMURE ${ }^{1}$, Keishi SUGA ${ }^{2}$, Hiroshi UMAKOSHI ${ }^{2}$, Jin MATSUMOTO ${ }^{1}$ \\ and Koichiro SHIOMORI ${ }^{{ }^{*}}$ \\ ${ }^{1}$ Department of Applied Chemistry, University of Miyazaki, 1-1 Gakuenkibanadai-nishi, Miyazaki 889-2192, \\ Japan; ${ }^{2}$ Division of Chemical Engineering, Graduate School of Engineering Science, Osaka University, 1-3 \\ Machikaneyama, Toyonaka, Osaka 560-8531, Japan
}

(Received January 7, 2017; Accepted April 5, 2017)

Poly- $N$-isopropylacrylamide (PNIPA) cryogels containing liposomes were prepared by polymerization under freezing conditions at sub-zero temperature. The cryogel had a highly porous structure with 20-30 $\mu \mathrm{m}$ pore size. The wall thickness of the cryogel containing liposomes was larger than that without liposomes. The structural change and aggregation of the liposomes in the cryogel were observed. The immobilization of the phospholipid and the dynamic property of the lipid membrane in the cryogel were confirmed. The cryogel containing liposomes showed chiral recognition ability toward L-Trp. The selective adsorption of L-Trp was achieved with the PNIPA cryogel containing liposome.

\section{Introduction}

Cryogels are macroporous polymer materials prepared under freezing conditions at sub-zero temperatures. Under freezing conditions, the reaction mixture separates into two phases, a solid ice phase and an unfrozen liquid micro-phase. In the micro-phase, polymerization of monomers or crosslinking of linear-chain polymers is carried out to form a hydrogel by the corresponding polymerization initiator or cross-linking reagent. After the reaction, the ice crystals in the reaction mixture are unfrozen at room temperature. In these processes, the hydrogel formed in the micro-phase forms a wall of cryogels and many interconnected macro-pores are formed to provide a supermacroporous structure [1-3]. The macroporous structure of the cryogels provides beneficial properties for water treatment, such as high water permeation without high backpressure and permeation of water containing particulate matter without clogging $[4,5]$. The preparation of the composite materials of cryogels with solid micro/nano-particles is also investigated [6-11]. Hence, cryogels are expected to provide for new filters and adsorbents in water treatment [3-11].

A liposome is a spherical molecular self-assembly of phospholipid molecules with a bilayer structure [12-14]. The liposome membrane has quite unique static and dynamic properties [15]. In recent years, it is reported that a liposome can recognize and selectively adsorb L-amino acids on its surface from the 
aqueous solution [16]. The recognition of L-/D-amino acids by a liposome is considered to be achieved through the combination of (i) electrostatic and (ii) hydrophobic interaction, and (iii) the stabilization effect of hydrogen bonds in the hydrophobic environment of the liposome membrane [16]. This recognition is similar to like as antigen recognition. Hence, the liposome is expected to be a chiral separation media for racemic compounds. The application of a liposome as separation medium and a reaction platform using its membrane properties are investigated by immobilization and integration with solid support materials to facilitate the handling of the liposome, which is suspended in water [18-21].

In this study, liposomes were immobilized in a poly- $N$-isopropyl acrylamide(PNIPA) cryogel. The liposomes used for immobilization were the commercially-available liposome suspensions to check the effect of the preparation conditions on the preparation and the morphology changes. The morphology of the cryogels containing liposomes, the membrane properties of the liposomes, and those immobilized in the cryogels were investigated. The adsorption of L/D-tryptophan using the cryogels containing liposomes were also investigated.

\section{Experimental}

\subsection{Materials}

$N$-isopropylacrylamide (NIPA), $N, N^{\prime}$-methylenebisacrylamide, ammonium persulfate, tetramethylethylenediamine, L-tryptophan (L-Trp) and D-Trp were supplied from Wako Pure Chemicals Industries, Ltd. (Japan). A commercially-available liposome suspension (EXTRASOME AQUA), which contains $5 \mathrm{wt} \%$ liposome in water and is about $100 \mathrm{~nm}$ in size, and 1,2-dipalmitoyl-sn-glycero-3phosphocholine (DPPC) were obtained from NOF Corporation (Japan).

\subsection{Preparation of Liposome}

DPPC (62.3 mg) was dissolved in $30 \mathrm{~mL}$ of chloroform. The chloroform solution of DPPC was dried in a round-bottomed flask under vacuum with a rotary evaporator to prepare a phospholipid thin film. The thin film was hydrated with distilled water $(1 \mathrm{~mL})$ at room temperature to prepare a vesicle suspension. The vesicle suspension was frozen at $-80{ }^{\circ} \mathrm{C}$ and thawed at $50{ }^{\circ} \mathrm{C}$ to enhance the transformation of small vesicles to large multilamellar vesicles (MLVs); this freeze-thaw cycle was performed five times. MLVs were used to prepare smaller unilamellar vesicles by extruding the MLV suspension 11 times through two layers of polycarbonate membranes, with mean pore diameters of $100 \mathrm{~nm}$, using an extruding device (Liposofast; Avestine Inc., Ottawa, ON, Canada). The unilamellar liposomes so obtained were concentrated by centrifugation at $135,000 \times g$ for $2 \mathrm{~h}$ at $4^{\circ} \mathrm{C}$. The prepared DPPC liposomes were used to compare the membrane properties with those of the commercial liposomes.

\subsection{Preparation of PNIPA cryogel containing liposome}

The preparation conditions for the cryogels containing commercially-available liposomes are shown in Table 1. $N$-isopropyl acrylamide as a monomer and $N, N$-methylene bis(acrylamide) as a cross-linker were dissolved in distilled water. The solution was blown with $\mathrm{N}_{2}$ gas in order to remove oxygen because 
oxygen inhibits the radical polymerization. With stirring under ice cooling, the liposome suspension together with ammonium peroxodisulfate as a polymerization initiator were added to the solution. After that, $N, N, N^{\prime}, N^{\prime}$-tetramethyl ethylene diamine was added to the solution and the solution mixture of $5 \mathrm{~mL}$ was poured immediately into $6 \mathrm{~mL}$ syringes and put in a cooling bath (CUL-MINI2, IWAKI) which was filled with ethanol and kept at $-15{ }^{\circ} \mathrm{C}$. After cooling overnight, the cryogels formed in the syringes were defrosted and washed with water. The gel preparation without freezing was also carried out by the same procedure and the polymerization took place at room temperature. The gel prepared without freezing is called a normal hydrogel.

Table 1. Preparation conditions for the cryogel containing liposomes

\begin{tabular}{cc}
\hline Reagent & Amounts \\
\hline$N$-Isopropyl acrylamide (NIPA) & $2.26 \mathrm{~g}$ \\
$N, N^{\prime}$-Methylenebisacrylamide (BAA) & $0.50 \mathrm{~g}$ \\
Ammonium persulfate (APS) & $0.25 \mathrm{~g}$ \\
$N, N, N^{\prime}, N^{\prime}$-Tetramethylethylenediamine (TEMED) & $60 \mu \mathrm{L}$ \\
Distilled water (free oxygen) & $25 \mathrm{~mL}$ \\
Liposome suspension (EXTRASOME AQUA) & $25 \mathrm{~mL}$ \\
\hline
\end{tabular}

\subsection{Scanning electron microscopy observation}

The cryogel was freezed by dipping in liquid nitrogen for $30 \min -1 \mathrm{~h}$, and dried using vacuum freeze drying equipment (VD-250, TAITEC, Ltd., Japan). The freeze-dried sample was sliced with a razor and plated by sputtering (E-1030, HITACHI, Japan), and examined using SEM (TM-1000, HITACHI, Japan).

\subsection{Transmission electron microscopy observation}

The freeze-dried cryogel was ground using a agate mortar. The powdered samples were diffused into ethanol and treated with ultrasonic irradiation for $5 \mathrm{~min}$. The dispersed samples were put on a carbon mesh, and examined using TEM (JEM-2010MX, JEOL, Ltd., Japan).

\subsection{Microscopic fluorescent image}

Octadecylrhodamine B, a fluorescent probe which is incorporated into the liposome membrane, was added to the liposomes suspensions at $0.1 \mathrm{~mol} \%$ for the phospholipid in the liposome. Microscopic fluorescence images of the liposome and the cryogels containing the liposomes were observed with an Olympus FV-300 confocal laser scanning microscope (CLSM, OLYMPUS Corporation, Tokyo, Japan) equipped with a x60 water immersion objective lens (numerical aperture $=1.2$ ). The wavelengths of the excitation laser/emission filter were $543 \mathrm{~nm} / 565 \mathrm{~nm}$. The cryogel containing liposomes that were labeled by the fluorescent probe was cross-sectioned as a thin sheet and also observed on a glass slide in the same manner as for the liposomes. 


\subsection{Investigation of the membrane properties using Raman spectroscopy}

The Raman spectra of the cryogel with and without liposomes and the liposome suspensions were recorded using a confocal Raman microscope (LabRAM HR-800, Horiba, Ltd., Kyoto, Japan) at $532 \mathrm{~nm}$, for a total data accumulation time of $20 \mathrm{~s}$. For each sample, the background signal of the solution was removed and then the baseline was corrected. The packing density of the lipid membrane, $R$, was determined using the following equation [17]:

$$
R=\frac{I_{2880}}{I_{2930}}
$$

where $I_{2880}$ and $I_{2930}$ are the peak intensities at 2880 and $2930 \mathrm{~cm}^{-1}$, respectively.

\subsection{Adsorption of tryptophan on a cryogel containing liposomes}

The cryogel $(0.20 \mathrm{~g})$ containing liposomes was put in distilled water $(30 \mathrm{~mL})$ containing $1.0 \mathrm{mM} \mathrm{L}$ or D-tryptophan (Trp), $C_{0}$, and incubated at $30^{\circ} \mathrm{C}$ for $66 \mathrm{~h}$. After incubation, the Trp solution was filtered with a membrane filter $\left(0.22 \mu \mathrm{m}\right.$ 、 Millex GS, Merck Japan). The concentration of Trp in the solution, $C_{\mathrm{W}}$, was measured from the absorbance at $280 \mathrm{~nm}$ using a UV spectrometer (UV-1200, Shimadzu corporation, Kyoto Japan). The adsorption concentration of Trp on the cryogel was obtained from the difference of $C_{0}$ and $C_{\mathrm{W}}$ [21]. The rate of adsorption of Trp was examined in the same manner. After the first adsorption, the cryogel was used again for the second adsorption time rate under the same condition.

\section{Results and Discussion}

\subsection{Observation of cryogel containing liposomes}

The visual and SEM observations of the PNIPA cryogel with and without liposomes are shown in Figure 1. The cross-section of the cryogels were observed with SEM. The cryogels had a porous structure, the size of pores were about 20-30 $\mu \mathrm{m}$. All cryogels prepared in this study showed relatively high water permeability without pressurization when they were set in the syringe tube. The wall thickness of the cryogel containing liposome was greater than that without liposomes. This increase in the wall thickness is caused by incorporation of liposomes into the cryogel wall.

The surfaces of the PNIPA cryogel without liposomes in the syringe used for the preparation were observed using SEM as shown in Figure 2. The cryogel without liposomes had a radiating structure in all

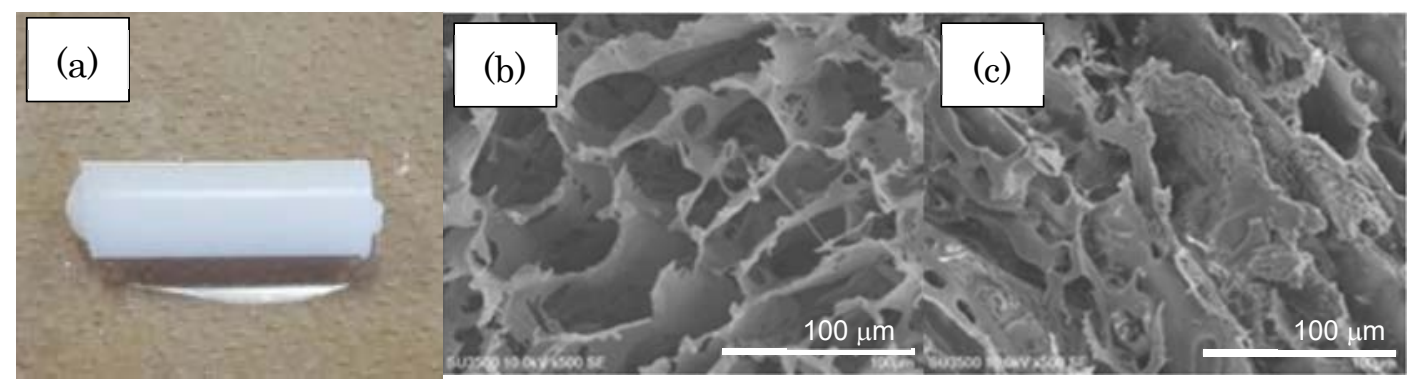

Figure 1. Visual and SEM observations of PNIPA cryogels, (a): cryogel containing liposomes, (b): SEM image of the cryogel without liposome, (c) SEM image of the cryogel containing liposomes. 
directions on the surfaces, particularly on the bottom surface. It is considered that this radiating structure is formed by the growth of ice crystals at the freezing step in the cryogel preparation. On the other hand, the cryogel containing liposomes was observed to have no radiating structure as shown in Figure 3. Both the cryogels containing and without liposome had an even sized porous structure at the side surface.

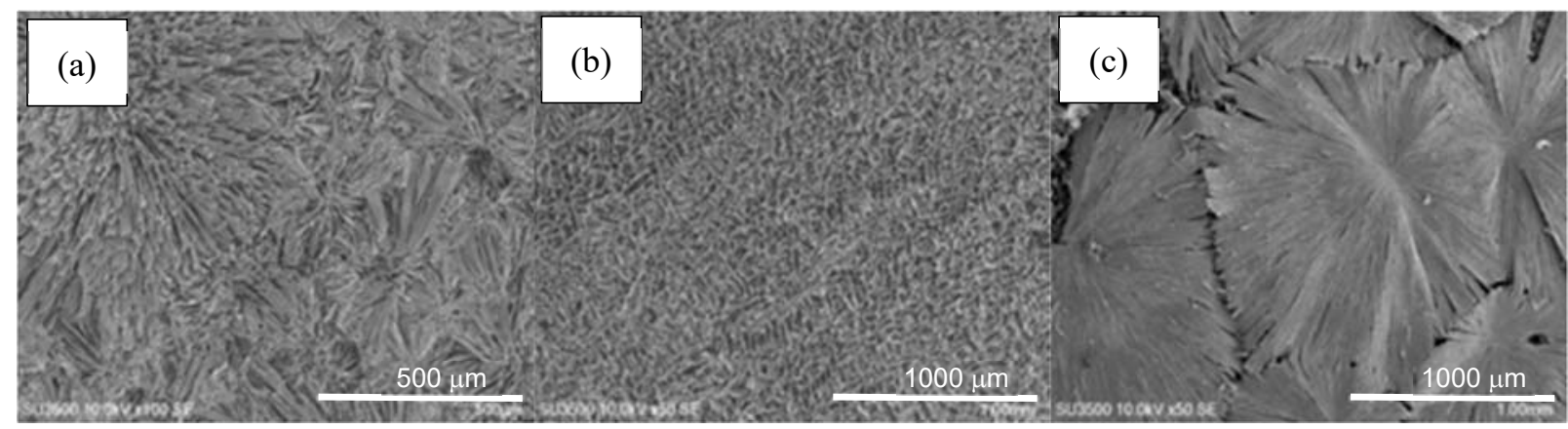

Figure 2. SEM images of a PNIPA cryogel without liposome, (a): top surface; (b): side surface; (c) bottom surface in the syringe.

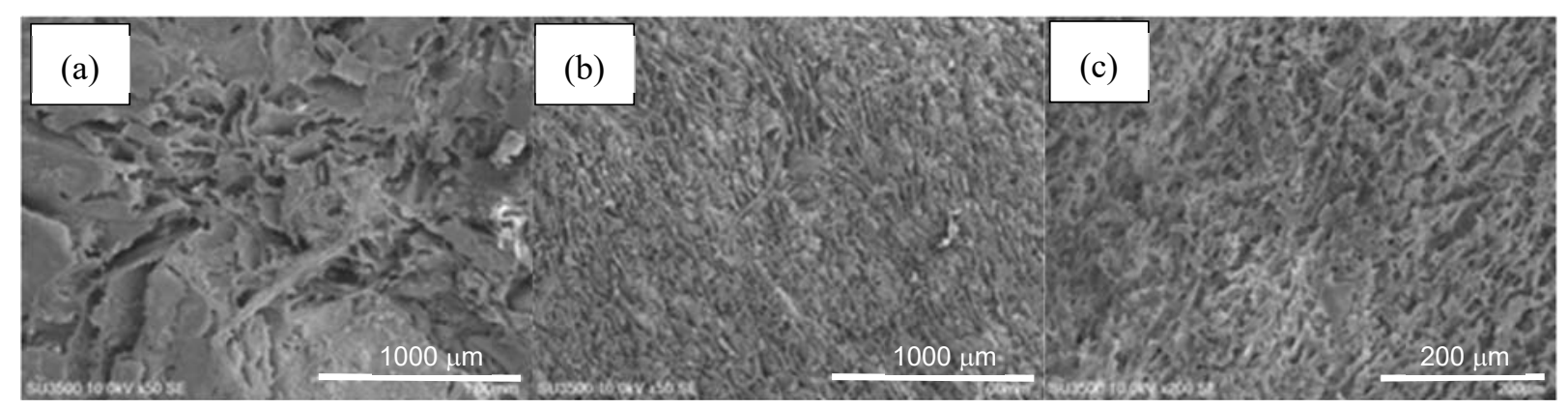

Figure 3. SEM images of PNIPA cryogel containing liposomes (a): top surface; (b): side surface; (c) bottom surface in the syringe.

Figure 4 shows the observation of the cryogel containing liposomes with TEM. Several spherical substances, of size $50-200 \mathrm{~nm}$, were confirmed in the cryogel wall as shown in Figure 4(a). In another part of the cryogel as shown in Figure 4(b), many aggregated spherical substances were observed and seemed to be unilamellar liposomes because the layered structure was observed as the wall of spherical

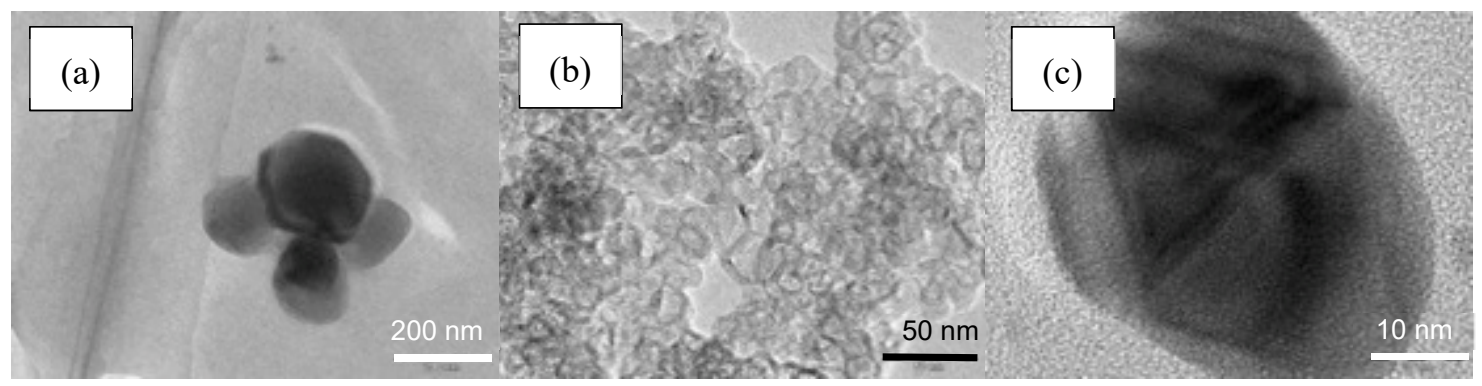

Figure 4. TEM images of PNIPA cryogel containing liposomes at various migrations. 
substances. Aggregated bilayer structures of greater size were also observed in the cryogel wall. The image of Figure 4(c) also suggested that the spherical substances were crystalline state of lipids because many linear images were observed on the surface. These results suggest the structure of the liposome would be changed during freezing and thawing in the cryogel preparation.

The liposome suspension used for the cryogel preparation and the prepared cryogel containing the liposomes, to which was added the fluorescent probe, was observed with a confocal laser scanning fluorescence microscope as shown in Figure 5. The fluorescence of octadecylrhodamine B shows the existence of a lipid bilayer structure [22]. In Figure 5(a), many fluorescent substances were observed of 1-2 $\mu \mathrm{m}$ size in the image and dispersed in the solution. This implies the existence of liposomes with a bilayer structure and a highly dispersed state in the suspension. Figure 5(b) shows the observation of the cryogel containing liposomes labeled with the fluorescent probe. Many fluorescent substances in a highly aggregated state were observed in the cryogel sample. This suggests the assembling and aggregation of liposomes and also the collapse of the spherical liposome structure. However, the strong fluorescence implies that the bilayer membrane structure is retained in the cryogel wall.

\subsection{Raman spectroscopy analysis of the cryogel containing liposome}

The liposome suspension used for the cryogel preparation and the prepared cryogel with and without the liposomes were analyzed using Raman spectrometry as shown in Figure 6. The common peak that arises from phospholipids at $2850 \mathrm{~cm}^{-1}$ was observed both in the cryogel containing liposomes and the liposome suspension. This shows the cryogel so prepared contains the phospholipid of liposome. Raman analyses of the cryogel containing liposome, the liposome suspension and also DMPC liposome were carried out at various temperatures to investigate the dynamic properties of the phospholipid membrane and the changes caused by the immobilization in the cryogel. Figure 7 shows the temperature dependencies of

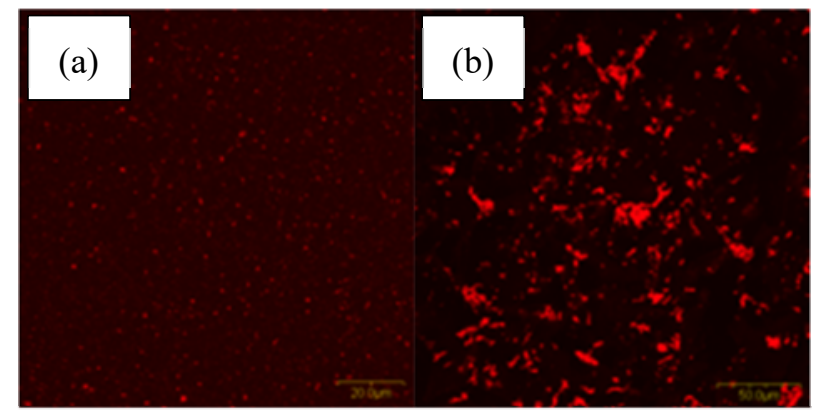

Figure 5. Confocal laser scanning microscope observation of the liposome suspension (a) and PNIPA cryogel containing liposomes (b) to which is added the fluorescent probe.
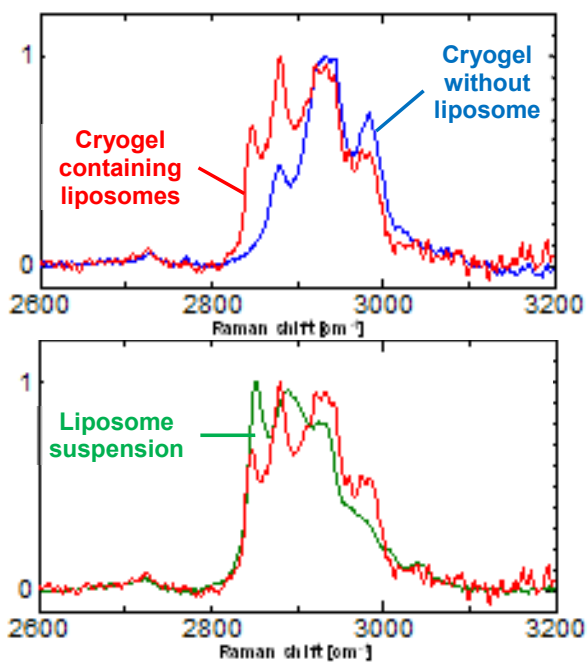

Figure 6. Raman spectroscopic analysis of the cryogel containing liposomes, the cryogel without liposome and the liposome suspension. 
the packing density of the lipid membranes calculated from Raman spectrum [16]. DPPC liposomes form the gel phase below the phase transition temperature at $45{ }^{\circ} \mathrm{C}$ with higher packing density values. The packing density decreased above $45{ }^{\circ} \mathrm{C}$. This shows the transition to the liquid-crystalline phase. The packing density of the liposome suspension, EXTRASOME AQUA, was almost the same value as that DPPC liposome below $45{ }^{\circ} \mathrm{C}$. This value slightly decreased above $45{ }^{\circ} \mathrm{C}$. The phospholipid membrane of the liposome suspension was suggested to have almost the same phase transition temperature as that for the DPPC liposome. On the other hand, the packing density of the liposome suspension immobilized in the cryogel had a low value suggesting that the dynamic properties of the lipid membrane of the liposome suspension was partially changed by the immobilization in the cryogel. This result corresponds with the aggregation in the fluorescence image observation.

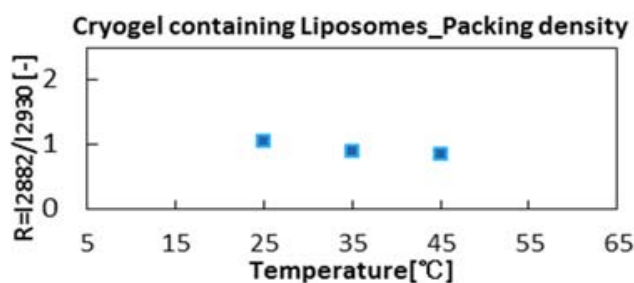

EXTRASOME AQUA_Packing density
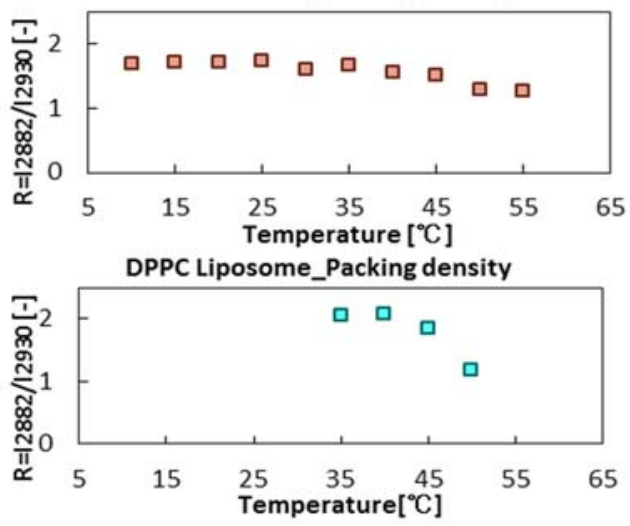

Figure 7. Temperature dependence of the packing density of the DPPC liposome membrane, the liposome suspension, and the liposome suspension immobilized in the cryogel.

\subsection{Adsorption behavior of tryptophan with the cryogel containing liposome}

The cryogel containing liposome was used for the adsorption of L-Trp and D-Trp. The rate of Trp adsorption with the cryogel containing liposome is shown in Figure 8(a). The adsorption concentration of Trp on the cryogel was plotted against the adsorption time. After $24 \mathrm{~h}$ of an induction period that shows no adsorption, the adsorbed concentration of L-Trp gradually increased with time. On the other hand, the adsorption of D-Trp was quite low. These results are similar to that for the hydrogel containing DMPC liposome [21]. It is clear that the commercially available liposome has the ability to recognize L-Trp and the chiral selective separation of tryptophan can be achieved using the cryogel containing liposomes prepared in this study. 
In order to clarify the adsorption properties of L-Trp, the cryogel containing liposome after adsorption for $66 \mathrm{~h}$ was put in a new aqueous solution containing L-Trp to restart the adsorption. The total adsorption concentration of L-Trp from the first adsorption was plotted against time in the second adsorption as shown in Figure 8(b). After the induction period, the adsorption concentration increased again with time. The induction period in the second adsorption was clearly shorter than that in the first adsorption. This suggests that the structure of the lipid bilayer in the cryogel would be changed effectively to fit the L-Trp adsorption during the first adsorption process after the long induction period [15-17, 21].
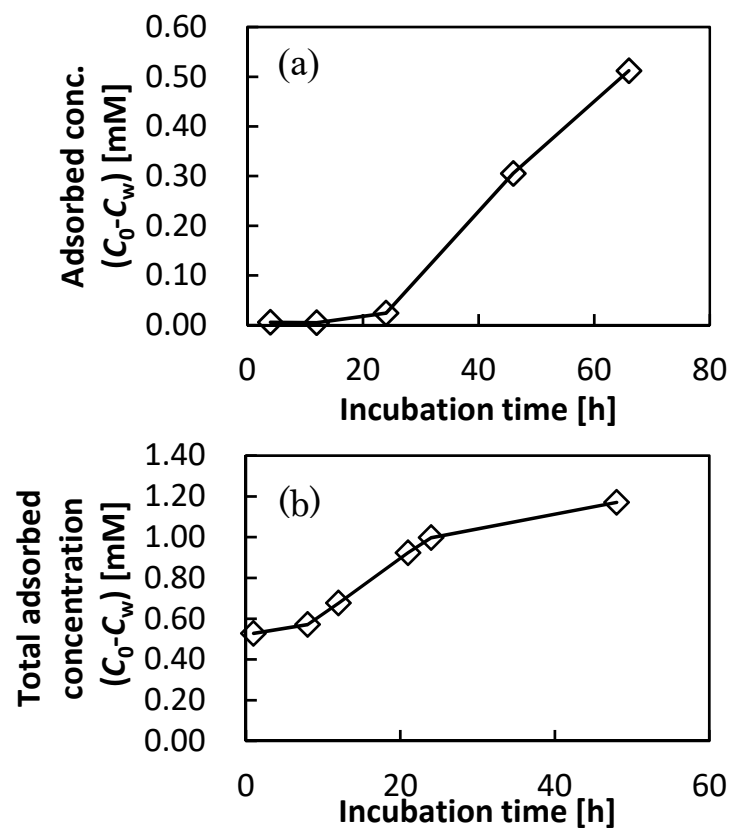

Figure 8. Rate of Trp adsorption with cryogel containing liposomes.

(a): First adsorption; (b): second adsorption. $C_{0}=1.0 \mathrm{mM}$.

The adsorption of L- and D-Trp was carried out using the cryogel and the normal hydrogel with or without liposome. The adsorbed concentration of Trp with the cryogel and the hydrogel are shown in Figure 9. The cryogel and the hydrogel containing liposomes adsorbed L-Trp, but the adsorption of D-Trp was negligible. The recognition of liposomes immobilized in the cryogel and the hydrogel for L-Trp was successfully observed. The difference between adsorption on the cryogel was small. This result suggests that the freezing process in the preparation of the cryogel did not affect the phospholipid membrane properties. Further investigation is necessary in order to clarify the changes of the structure and the dynamic properties of the liposome immobilized in the cryogel, which strongly participates in the chiral recognition ability of the liposome. 

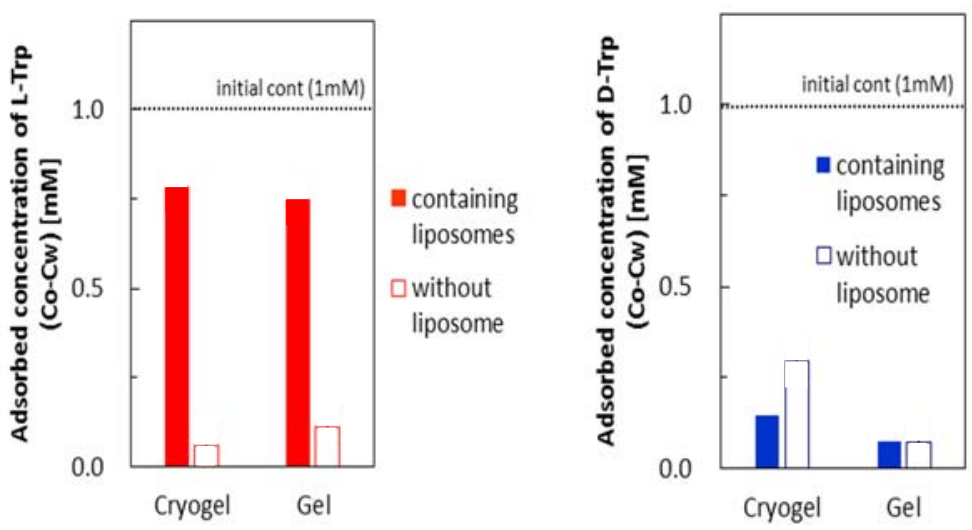

Figure 9. Adsorbed concentration of L-Trp and D-Trp with cryogel and hydrogel (Gel) containing or without liposomes after $66 \mathrm{~h}$ incubation.

\section{Conclusion}

A cryogel containing a commercially-available liposomes suspension was successfully prepared with a highly porous structure. The pore size in the cryogel was about $20-30 \mu \mathrm{m}$. The wall thickness of the cryogel containing liposomes was larger than that without liposomes. The structural change and aggregation of liposomes in the cryogel were observed from the TEM and fluorescence microscope image analyses. The common peak detected in the cryogel containing liposomes and the liposome suspension by Raman spectrometry showed the immobilization of the phospholipid in the cryogel. The packing density of the phospholipid membrane was decreased by the immobilization of the liposome suspension in the cryogel and showed the change in the dynamic properties of the lipid membrane. The cryogel containing liposomes showed chiral recognition ability toward L-Trp and exhibited the selective adsorption of L-Trp.

\section{Acknowledgement}

This research was supported by a Japan Society for the Promotion of Science (JSPS) Grad-in-Aid for Scientific Research A (No. 26249116) and partly a JSPS Grant-in-Aid for Exploratory Research (No. 26550063).

\section{References}

1) F. M. Plieva, M. Karlsson, M. R. Aguilar, D. Gomez, S. Mikhalovsky, I. Y. Galaev, Soft Matter, 1, 303-309 (2005).

2) F.M. Plieva, M. Karlsson, M.-R. Aguilar, D. Gomez, S. Mikhalovsky, I.Yu. Galaev, B. Mattiasson, J. Appl. Polym. Sci. 100, 1057-1066 (2006).

3) V.I. Lozinsky, Adv. Polym. Sci., 263, 1-48 (2014). 
4) G. Ertürk, B. Mattiasson, J. Chromatography A, 1357, 24-35 (2014).

5) A. Kumar, A. Srivastava, Nature Protocols, 5, 1737-1747 (2010).

6) I. N. Savina, C. J. English, R. L. D. Whitby, Y. Zheng, A. Leistner, S. V. Mikhalovsky, A. B. Cundy, J. Hazard. Materials, 192, 1002-1008 (2011).

7) L. Önnby, V. Pakade, B. Mattiasson, H. Kirsebom, Water Res., 46, 4111-4120 (2012).

8) A.E. Ivanov, O.P. Kozynchenko, L.I. Mikhalovska, S.R. Tennison, H. Jungvid, V.M. Gun'ko, S.V. Mikhalovsky, Phys. Chem. Chem. Phys., 14, 16267-16278 (2012).

9) M. Kurozumi, Y. Yano, S. Kiyoyama, A. Kumar, K. Shiomori, Resources Processing, 62, 17-23 (2015).

10) M. Kurozumi, Y. Yano, S. Kiyoyama, A. Kumar, K. Shiomori, Stud. Sci. Technol., 5, $23-28$ (2016).

11) A. E. Ivanov, T. Halthur, L. Ljunggren, J. Environ. Chem. Eng., 4, 1432-1441 (2016).

12) P. Yeagle, in "The Structure of Biological Membranes, 3rd Ed." (CRC Press), Chapter 4, 19-91 (2011).

13) C. Huang, J. T. Mason, I. W. Levin, Biochemistry, 22, 2775-2780 (1983).

14) R. L. Hamilton, Jr, J. Goerke, L. S. Guo, M. C. Williams, R. J. Havel, J. Lipid Res., 21, 981-992 (1980).

15) H. Umakoshi, K. Suga, Solvent Extr. Res. Dev. Jpn., 20, 1-13 (2013).

16) T. Ishigami, H. Umakoshi, Solvent Extr. Res. Dev. Jpn., 20, 213-217 (2013).

17) T. Ishigami, K. Suga, H. Umakoshi, ACS App. Matr. Interfaces, 7, $21065-21072$ (2015).

18) Q. Yang, M. Wallstén, P. Lundahl, Biochim. Biophys. Acta-Biomembranes, 938, 243-256 (1988).

19) H. Sugaya, H. Umakoshi, Y. Tohtake, T. Shimanouchi, R. Kuboi, Solvent Extr. Res. Dev. Jpn., 16, 103-109 (2009).

20) Y. Liu, Z. Li, D. Liang, Soft Matter, 8, 4517-4523 (2012).

21) T. Ishigami, K. Sugita, K. Suga, Y. Okamoto, H. Umakoshi, Colloid Surface B, 136, 256-261 (2015).

22) J. Mastumoto, K. Yoneda, J. Tasaka, M. Yasuda, Bull. Chem. Soc. Jpn., 83, 1551-1557 (2010). 